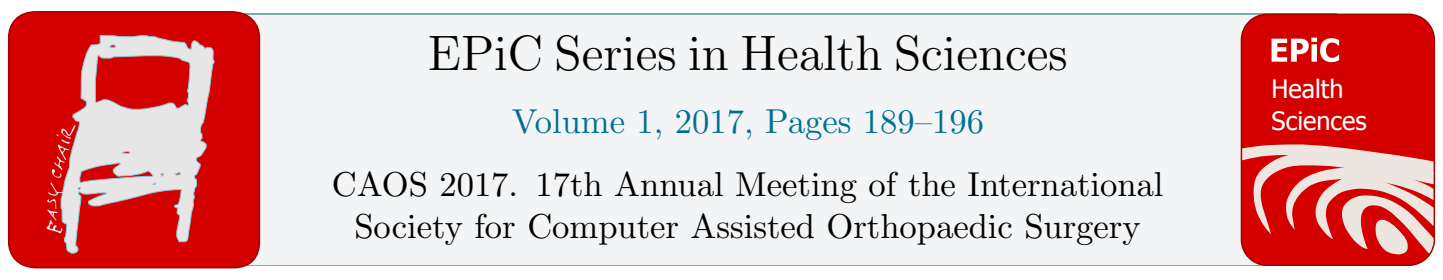

\title{
A Smart Registration Assistant for Joint Replacement: Concept Demonstration
}

\author{
He Liu ${ }^{1 *}$, Stuart Bowyer ${ }^{1}$, Edouard Auvinet ${ }^{2}$ and Ferdinando Rodriguez y \\ Baena $^{1 \dagger}$ \\ ${ }^{1}$ Mechatronics in Medicine Lab, Imperial College, London, U.K. \\ ${ }^{2}$ MSk Lab, Imperial College, London, U.K. \\ he.liul5@imperial.ac.uk, f.rodriguez@imperial.ac.uk
}

\begin{abstract}
In robot-assisted orthopaedic surgery, registration is a key step, which defines the position of the patient in the robot frame so that the preoperative plan can be performed. Current registration methods have their limitations, such as the requirement of immobilising the limb or maintaining the line of sight (LOS). These issues cause inconvenience for the surgeons and interrupt the surgical workflow in the operating room.

Targeting these issues, we propose a smart camera-robot registration system for joint replacement. The bone geometry, which is measured directly by a depth camera, is aligned to a preoperatively obtained bone model to calculate the pose of the target. Simultaneously, in order to avoid registration failure caused by LOS disruptions, the depth camera tracks objects that may occlude the target bone, and a robot manipulator is used to move the camera away from the nearest obstacle. An appropriate camera motion to "escape" the obstacle is calculated based on the position and velocity of the obstacle, with the aim of avoiding the occlusion efficiently without changing the general target position in the camera frame. The inverse kinematics of the robot is used to project the Cartesian velocity of the end-effector into the joint space, with kinematic singularities considered for stable robotic control. An admittance controller is designed as the human-robot interface so that the surgeon can directly set the robot configuration by hand according to a given intraoperative scenario.

Simulations and experiments with a redundant manipulator were conducted to test the performance of a proof-of-concept implementation. The results show that the proposed obstacle avoidance method can effectively increase the distance between the obstacle and the LOS, which lowers the risk of registration failure due to obstacle occlusion. This pilot study is promising in reducing distractions to the surgeon and could help achieve a fluent and surgeon-centred workflow.
\end{abstract}

${ }^{*}$ Created the first draft of this paper

${ }^{\dagger}$ Principal investigator 


\section{Introduction}

Among all the orthopaedic procedures, joint replacement, which mainly includes hip and knee replacement, is the most common one. Targeting the issues of limited surgical accuracy and inconvenient preoperative planning of conventional joint replacement, robotic assistance has been integrated into the operating room (OR), such as with ROBODOC (Curexo Tech. Corp.), RIO (Stryker Corp.) and Navio PFS (Smith and Nephew Plc.), which have led to improved surgical outcomes (Lang, et al., 2011) (Netravali, Shen, Park, \& Bargar, 2013) (Koenig \& Hepinstall, 2015). A key step for robot-assisted joint replacement is registration, which establishes the spatial alignment of the robot frame, the patient and the preoperative plan (Lea, et al., 1995). Registration methods currently in use are generally categorised as image-based or image-free methods. Image-free methods require user interaction for landmark identification, and their accuracy tends to be localised (Sugano, 2013); image-based methods suffer from additional preoperative time, line of sight (LOS) issues and may require immobilisation of the limbs (Maintz \& Viergever, 1998) (Koenig \& Hepinstall, 2015).

Because of their simplicity and accuracy, depth cameras are becoming increasingly popular in the field of robotics. In contrast to conventional stereo cameras, depth cameras directly utilise light techniques, such as structured-light and time-of-flight, to measure 3D positions actively and directly (Langmann, Hartmann, \& Loffeld, 2012). With the development of more accurate and faster depth measurement abilities, depth cameras show great potential in orthopaedic registration. However, the potential loss of LOS, which is a significant limitation of optical trackers during registration, remains a problem. Additionally, aspects of human-robot coexistence within a cluttered OR still remain. Noncompliant robots tend to take up a large workspace in the OR and may cause distraction to the surgeon, thus interrupting the surgical workflow.

Targeting these problems, the aim of this research is to develop a smart registration assistant for robot-assisted joint replacement surgery that is self-adjustable, interactive and safe. To this end, a robot-camera registration system is presented here that combines the mobility of a robot and the sensing ability of a depth camera. The camera, which is mounted on, and calibrated to, the endeffector of the robot, tracks the target bone and obstacles that can potentially infringe on the camera's LOS, simultaneously. Desired camera motions are planned based on the motion of the obstacles, which are achieved by the robot through inverse kinematics, with singularities and joint limitations considered to guarantee stability. Additionally, an admittance controller is designed for the robot so that the surgeon can interact with it "hands on", in an intuitive way, according to the actual scenario. Laboratory experiments were conducted to verify the integration of registration and robotic control.

\section{Materials and Methods}

The smart registration system includes a seven degree-of-freedom (DOF) robotic manipulator and a depth camera. The depth camera is used for registration by scanning the environment to store spatial measurements in the form of a point cloud. A model-based registration method is adopted to process this point cloud to obtain the pose of the target bone. The registration method contains two steps: coarse registration and fine registration. Coarse registration is feature-based, and encompasses the computation of feature descriptors from 3D points to estimate a rough alignment of the target within the environment. This alignment is then used as the initial pose estimation for the fine registration, a vanilla implementation of the iterative closest point (ICP) algorithm (Besl \& McKay, 1992), which computes the accurate pose of the target in the camera coordinates. In order that the robot can use this spatial information, a calibration method (Sciavicco \& Siciliano, 2012) is adopted to obtain the relationship between the coordinates of the robot and the camera. 
As the ICP algorithm is iterative and only locally optimum, smaller changes between two sampled point clouds from subsequent frames will facilitate convergence of the algorithm to the same global minimum. Therefore, after the target is registered, the motion control strategy is to move the camera around the target while keeping the target at the same position in the camera coordinates. Based on the position of the target, an obstacle-free conical space is defined, with the cone's apex being the centre of the target, and its axis defined by the straight line between the target and the camera, as shown in Figure 1. The space should contain no obstacles, and so the camera will move away from one, if it is identified within this volume. For a static or slow obstacle, the camera simply moves in the opposite direction to the obstacle's incoming vector. Conversely, when the obstacle moves faster than the robot, a different motion plan is needed.

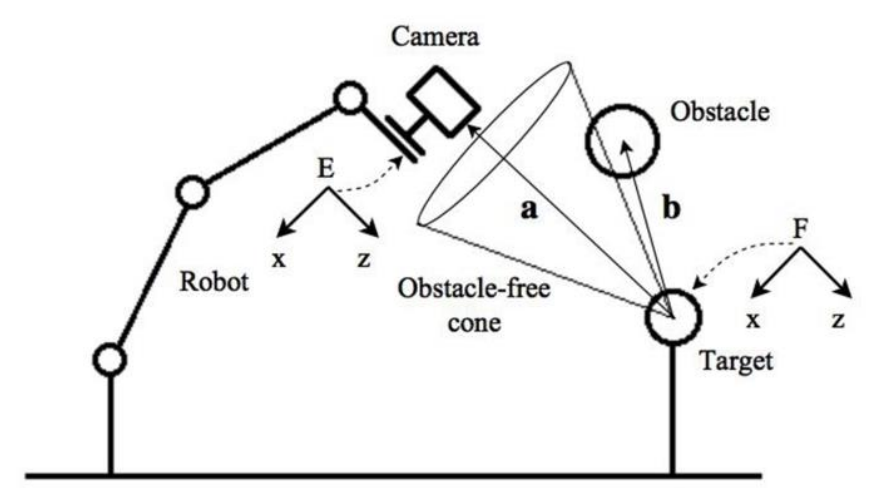

Figure 1: Spatial relationship between camera, target and obstacle.

Let $\mathbf{a}$ and $\mathbf{b}$ be the vectors from the target to the camera and the obstacle. To minimise the possibility of occlusion, the camera should move in the direction that keeps a away from $\mathbf{b}$. The angle between $\mathbf{a}$ and $\mathbf{b}$ is $\angle a b=\arccos (\hat{\mathbf{a}} \cdot \hat{\mathbf{b}})$, where $\hat{\mathbf{a}}, \hat{\mathbf{b}}$ are the unit vectors of $\mathbf{a}$ and $\mathbf{b}$. The arccosine function is monotone when $0 \leq \angle \mathrm{ab}<90^{\circ}$, so we only need to analyse $\hat{\mathbf{a}} \cdot \hat{\mathbf{b}}$. Let $\hat{\mathbf{k}}_{\mathrm{a}}, \hat{\mathbf{k}}_{\mathrm{b}}$ be the unit vectors defining the rotation axes of $\mathbf{a}$ and $\mathbf{b}$ as the camera and obstacle move, and $\omega_{\mathrm{a}}, \omega_{\mathrm{b}}\left(\omega_{\mathrm{a}}<\omega_{\mathrm{b}}\right)$ the angular velocities, then

$$
\frac{\partial}{\partial t}(\hat{\mathbf{a}} \cdot \hat{\mathbf{b}})=\frac{\partial \hat{\mathbf{a}}}{\partial t} \cdot \hat{\mathbf{b}}+\hat{\mathbf{a}} \cdot \frac{\partial \hat{\mathbf{b}}}{\partial t}=(\hat{\mathbf{b}} \times \hat{\mathbf{a}}) \cdot\left(\omega_{\mathrm{b}} \hat{\mathbf{k}}_{\mathrm{b}}-\omega_{\mathrm{a}} \hat{\mathbf{k}}_{\mathrm{a}}\right)
$$

$\omega_{\mathrm{b}} \hat{\mathbf{k}}_{\mathrm{b}}-\omega_{\mathrm{a}} \hat{\mathbf{k}}_{\mathrm{a}}$ is the velocity of $\mathbf{b}$ relative to $\mathbf{a}$, and $\hat{\mathbf{b}} \times \hat{\mathbf{a}}$ is the direction of $\mathbf{b}$ moving towards $\mathbf{a}$. Figure 2 shows the plane that contains $\mathbf{b} \times \mathbf{a}$ and $\omega_{\mathrm{b}} \hat{\mathbf{k}}_{\mathrm{b}}$. When the obstacle is approaching $\mathbf{a}$ with a speed faster than the camera, i.e. the projection of $\omega_{\mathrm{b}} \hat{\mathbf{k}}_{\mathrm{b}}-\omega_{\mathrm{a}} \hat{\mathbf{k}}_{\mathrm{a}}$ on $\mathbf{b} \times \mathbf{a}$ is positive, the angle between $\omega_{\mathrm{b}} \hat{\mathbf{k}}_{\mathrm{b}}-\omega_{\mathrm{a}} \hat{\mathbf{k}}_{\mathrm{a}}$ and $\mathbf{b} \times \mathbf{a}$ should be maximised. Thus, the optimal $\hat{\mathbf{k}}_{\mathrm{a}}$ is obtained by rotating $\hat{\mathbf{k}}_{\mathrm{b}}$ angle $\alpha$ anticlockwise, which can be calculated by the Rodriguez's rotational formula:

$$
\hat{\mathbf{k}}_{\mathrm{a}}=\hat{\mathbf{k}}_{\mathrm{b}} \cos (\alpha)+\left(\hat{\mathbf{k}} \times \hat{\mathbf{k}}_{\mathrm{b}}\right) \sin (\alpha)+\hat{\mathbf{k}}\left(\hat{\mathbf{k}} \cdot \hat{\mathbf{k}}_{\mathrm{b}}\right)(1-\cos (\alpha))
$$

where $\mathbf{k}=\mathbf{k}_{\mathbf{b}} \times(\mathbf{b} \times \mathbf{a}), \quad \alpha=\arccos \left(\omega_{\mathrm{a}} / \omega_{\mathrm{b}}\right)$. This $\hat{\mathbf{k}}_{\mathrm{a}}$ defines the Cartesian velocity of the robot's end-effector. Note that this motion planning is optimal only when $\omega_{\mathrm{b}}$ is constant, but the effect of acceleration is not significant. 


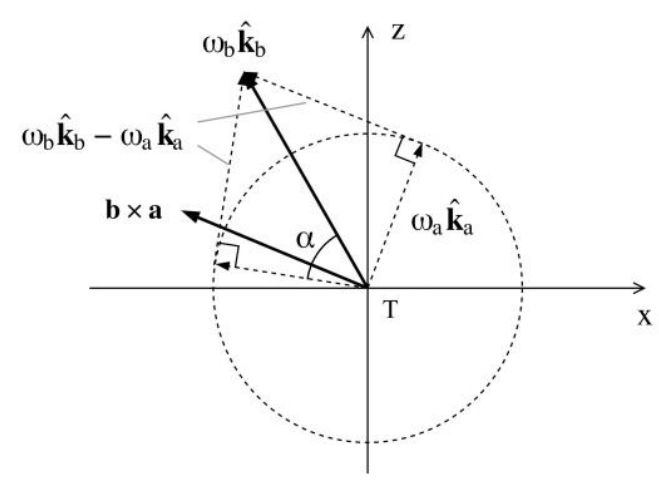

Figure 2: Motion planning for optimal obstacle avoidance. The radius of the dashed circle is $\omega_{\mathrm{a}}$.

After motion planning in Cartesian space, the velocity is transformed into joint space for robot control. As the position of the target bone is known to the robot after registration, an additional coordinate system $\{\mathbf{F}\}$ is added in the end-effector frame $\{\mathbf{E}\}$, with its origin coinciding with the target bone, as shown in Figure 1. The velocity relationship between $\{\mathbf{E}\}$ and $\{\mathbf{F}\}$ is

$$
\left[\begin{array}{c}
\mathbf{v}_{\mathrm{f}} \\
\boldsymbol{\omega}_{\mathrm{f}}
\end{array}\right]=\left[\begin{array}{c}
\mathbf{v}_{\mathrm{e}}+\boldsymbol{\omega}_{\mathrm{e}} \times \mathbf{p} \\
\boldsymbol{\omega}_{\mathrm{e}}
\end{array}\right]=\left[\begin{array}{cc}
\mathbf{I} & {[\mathbf{p}]_{\times}^{\mathrm{T}}} \\
\mathbf{0} & \mathbf{I}
\end{array}\right]\left[\begin{array}{c}
\mathbf{v}_{\mathrm{e}} \\
\boldsymbol{\omega}_{\mathrm{e}}
\end{array}\right]
$$

where $\mathbf{v}_{\mathrm{f}}, \boldsymbol{\omega}_{\mathrm{f}}$ and $\mathbf{v}_{\mathrm{e}}, \boldsymbol{\omega}_{\mathrm{e}}$ are the linear and angular velocities of $\{\mathbf{F}\}$ and $\{\mathbf{E}\}$ respectively, $\mathbf{I}$ is the identity matrix, $\mathbf{p}$ is the position vector of $\mathrm{F}$ in $\{\mathbf{E}\},[\mathbf{p}]_{x}^{T}$ is a skew-symmetric matrix and $[\mathbf{p}]_{\mathrm{x}}^{\mathrm{T}} \boldsymbol{\omega}_{\mathrm{e}}=\boldsymbol{\omega}_{\mathrm{e}} \times \mathbf{p}$. From (3), we have the forward kinematics of the robot:

$$
\dot{\mathbf{x}}=\left[\begin{array}{c}
\mathbf{v}_{\mathrm{f}} \\
\boldsymbol{\omega}_{\mathrm{f}}
\end{array}\right]=\left[\begin{array}{cc}
\mathbf{I} & {[\mathbf{p}]_{\mathrm{x}}^{\mathrm{T}}} \\
\mathbf{0} & \mathbf{I}
\end{array}\right] \cdot \mathbf{J} \dot{\mathbf{q}}=\mathbf{J}_{\mathrm{m}} \dot{\mathbf{q}}
$$

where $\dot{\mathbf{q}}$ is the robot's velocity vector in joint space.

For a redundant robot, we adopt the Moore-Penrose pseudoinverse (Moors, 1920) to calculate the inverse kinematics:

$$
\dot{\mathbf{q}}=\mathbf{J}_{\mathrm{m}}^{+} \dot{\mathbf{x}}+\left[\mathbf{I}-\mathbf{J}_{\mathrm{m}}^{+} \mathbf{J}_{\mathrm{m}}\right] \dot{\mathbf{q}}_{0}
$$

where $\mathbf{J}_{\mathrm{m}}^{+}=\mathbf{J}_{\mathrm{m}}^{\mathrm{T}}\left(\mathbf{J}_{\mathrm{m}} \mathbf{J}_{\mathrm{m}}^{\mathrm{T}}\right)^{-1}$, and $\dot{\mathbf{q}}_{0}$ is an arbitrary joint velocity vector. $\mathbf{I}-\mathbf{J}_{\mathrm{m}}^{+} \mathbf{J}_{\mathrm{m}}$ is the projection matrix to the null space of $\mathbf{J}_{\mathrm{m}}$, which means that after projection $\dot{\mathbf{q}}_{0}$ does not change the Cartesian velocity, so it can be used to achieve other tasks without violating the primary task. Here the redundant DOFs are utilised to avoid configuration singularities.

To use this robot-camera system with a "human in the loop", admittance control is adopted for human-robot interaction. This allows the surgeon to manually adjust the configuration of the robot according to the environment, thus improving safety. To manipulate the robot comfortably and intuitively, the surgeon is allowed to use two hands on the robot (which would be duly draped to guarantee sterility of the OR) to apply forces on its body. Thus, admittance is set for each joint of the robot rather than the end-effector. Forces on the robot are measured as a torque vector $\boldsymbol{\tau}$ (obtained via torque sensors within each link pair) that contains torques for all the joints. For each joint, its velocity is calculated as 


$$
\dot{\mathbf{q}}(i)=\boldsymbol{\tau}(i) \cdot \mathbf{Y}(i)
$$

where $\mathbf{Y}$ is the joint admittance vector, and $i=1 \ldots n$, where $n$ is the number of joints. In this simple proof of concept implementation, the joints are modelled as pure dampers with constant admittance values in order to avoid inertial effects after external forces are removed, and the admittance values are optimised empirically through experiments. Alternative dynamic admittance models are to be investigated and analysed in the future.

The admittance velocity can generate arbitrary motion of the end-effector. In order not to lose sight of the target, the robot motion is regulated like the obstacle avoidance motion, i.e. the camera moves on a sphere around the target. From (4) we have

$$
\dot{\mathbf{x}}=\left[\begin{array}{c}
\mathbf{v}_{\mathrm{f}} \\
\boldsymbol{\omega}_{\mathrm{f}}
\end{array}\right]=\mathbf{J}_{\mathrm{m}} \dot{\mathbf{q}}=\left[\begin{array}{c}
\mathbf{J}_{\mathrm{m} 1} \\
\mathbf{J}_{\mathrm{m} 2}
\end{array}\right] \dot{\mathbf{q}}
$$

where $\mathbf{J}_{\mathrm{m} 1}$ and $\mathbf{J}_{\mathrm{m} 2}$ project the joint velocities into Cartesian linear and angular velocities respectively. Therefore, the admittance joint velocity is projected to the null space of $\mathbf{J}_{\mathrm{m} 1}$ and no Cartesian linear velocity will be generated. The actual admittance velocity is:

$$
\dot{\mathbf{q}}_{\mathrm{a}}=\mathbf{P} \cdot \dot{\mathbf{q}}=\mathbf{P} \cdot \mathbf{T} \cdot \mathbf{Y}
$$

where $\mathbf{P}=\mathbf{I}-\mathbf{J}_{\mathrm{m} 1}^{+} \mathbf{J}_{\mathrm{m} 1}$ is the null space projector of $\mathbf{J}_{\mathrm{m} 1}, \mathbf{T}$ is an $n \times n$ diagonal matrix with the main diagonal being the external torque vector $\tau$.

\section{Results and Discussion}

A simulation was conducted in Matlab R2016a (the MathWorks Inc.) with the Robotics Toolbox (www.petercorke.com) to test different motion planning methods. The kinematic model of the robot was built using appropriate functions within the toolbox. The end-effector velocity for the robot

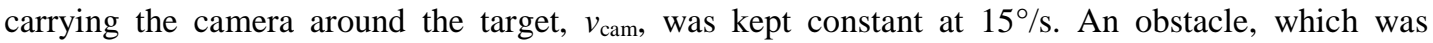
simplified here to be a point, was made to traverse the obstacle-free cone at different distances from the cone's principal axis, a, in Figure 1. The apex angle of the cone was set to $30^{\circ}$. Four groups of tests were performed, with the obstacle velocity ( $\left.v_{\text {obs }}\right)$ set at $1.2 v_{\text {cam }}, 1.5 v_{\text {cam }}, 2 v_{\text {cam }}$ and $3 v_{\text {cam }}$, and the simulation update rate was $100 \mathrm{~Hz}$. The angle between the target-camera vector, a, and the targetobstacle vector, b, was measured and the results are plotted in Figure 3.

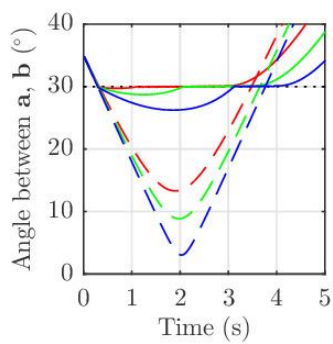

(a) $v_{\text {obs }}=1.2 v_{\text {cam }}$

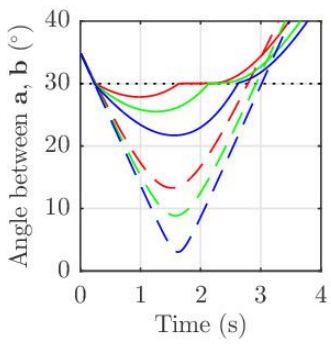

(b) $v_{\mathrm{obs}}=1.5 v_{\mathrm{cam}}$

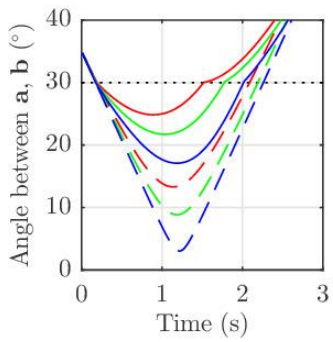

(c) $v_{\text {obs }}=2 v_{\text {cam }}$

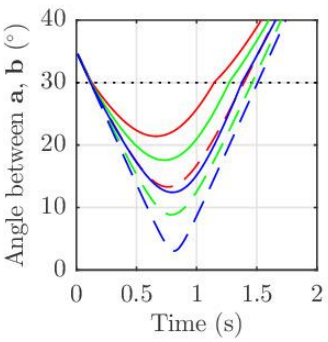

(d) $v_{\text {obs }}=3 v_{\text {cam }}$

Figure 3: Simulation results for the obstacle avoidance method. The dashed lines represent the results with the camera stationary. The red, green and blue solid lines represent obstacle avoidance trajectories of the camera as the obstacle traverses the obstacle-free cone. 
From Figure 3, when the obstacle velocity is not significantly larger than that of the camera (see Figure 3a-b), the obstacle avoidance method can effectively reduce penetration of the obstacle within the obstacle-free cone by more than $2 / 3$, and is almost entirely effective if the obstacle trajectory crosses the cone at some distance from a (Figure 3, red lines). With the obstacle velocity increasing (see Figure 3c-d), the avoidance results become less significant, although the obstacle is kept at a safe distance from the LOS, minimising the possibility of LOS disruption.

Experiments were subsequently conducted to test the obstacle avoidance performance. The depth camera used was the Xtion PRO LIVE (ASUSTeK Computer Inc.), mounted on a 7-DOF manipulator (LBR iiwa, KUKA Aktiengesellschaft). A second robot (LWR4+, KUKA Aktiengesellschaft) was used as the obstacle that moved along a planned trajectory. As here the obstacle was not a point, the point on the end-effector that was closest to the LOS was chosen as the input to the obstacle avoidance algorithm. Four tests were carried out, with the obstacle velocity set at $0.1 \mathrm{~m} / \mathrm{s}, 0.2 \mathrm{~m} / \mathrm{s}, 0.3$ $\mathrm{m} / \mathrm{s}$ and $0.4 \mathrm{~m} / \mathrm{s}$. Figure 4 shows the end-effector motion of the two robots during the experiments, while the distance between the obstacle and the LOS is plotted in Figure 5.

As can be seen from Figure 4 and 5, with the obstacle avoidance method, the camera was moved to the position with the least impaired LOS when an obstacle appears inside the defined cone. For slow moving obstacles, the distance to the LOS almost doubles compared with results obtained with the static camera configuration, resulting in a significantly reduced sight occlusion. However, as the speed of the obstacle increases, the average distance between the LOS and obstacle reduces, illustrating the limits of the current implementation. The avoidance results are thus not that effective for fast obstacles, but the best obstacle-free LOS is still achieved for a given set of robot, obstacle and camera parameters.

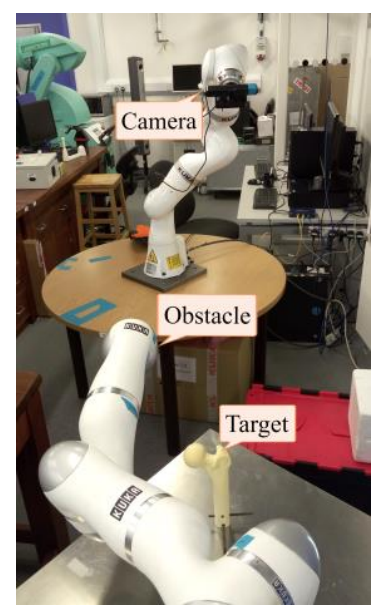

(a)

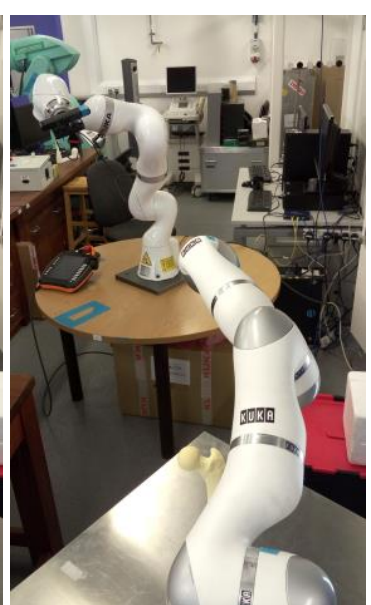

(b)

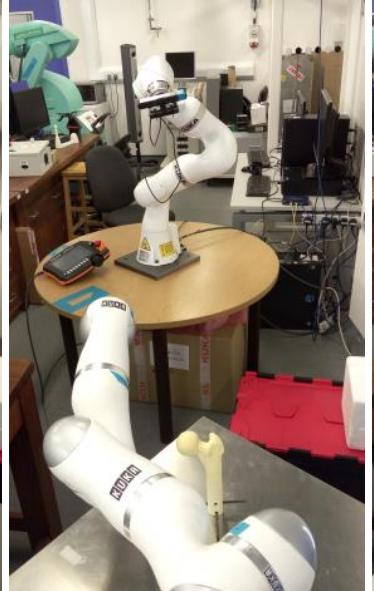

(c)

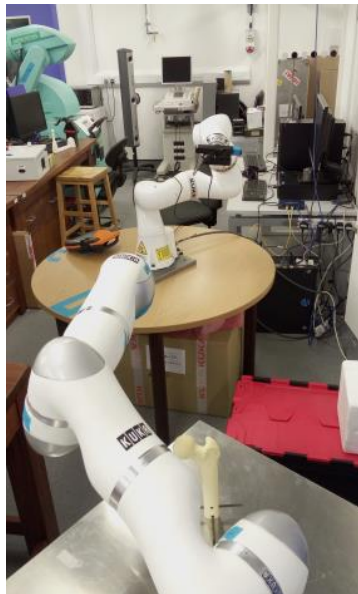

(d)

Figure 4: Experiment of obstacle avoidance with the robots in different configurations (a-d). 


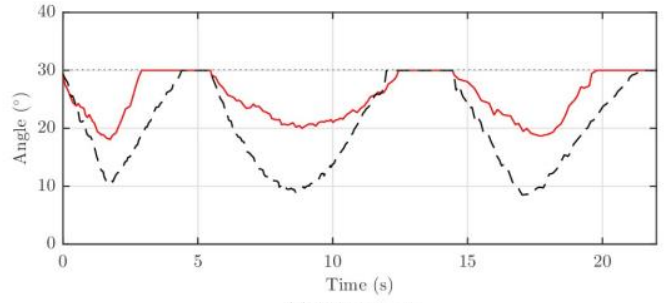

(a) Velocity $=v$

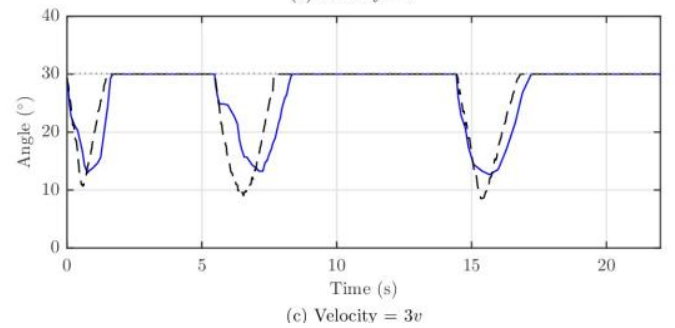

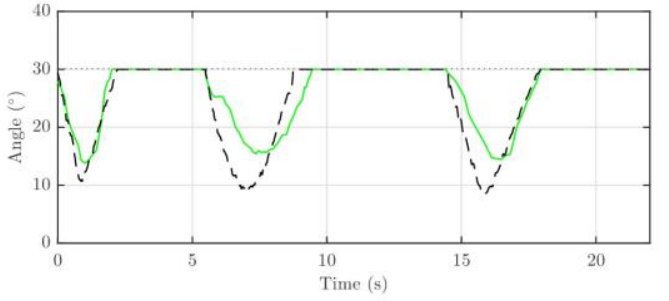

(b) Velocity $=2 v$

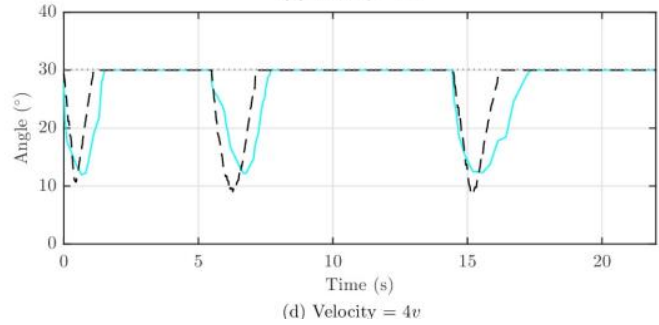

Figure 5: Experimental results for four different obstacle velocities (black dashed lines, a-d) against the robot escape trajectories (coloured lines, a-d). The black dotted line at $30^{\circ}$ illustrates the outer perimeter of the obstacle-free cone, outside which the camera stops tracking.

\section{Conclusion}

Robot-assisted orthopaedic surgery has proven its ability to improve accuracy and reduce outliers, but the operating time increases due to technical complexity and workflow interruptions (Goradia, 2014) (Karthik, Colegate-Stone, Dasgupta, Tavakkolizadeh, \& Sinha, 2015). To integrate robotic technology naturally into the OR with intuitive interfaces and minimal interference to the workflow, we propose a registration assistant utilising a depth camera for registration, while simultaneously avoiding potential obstacles in sight, which shows potential in reducing the registration failure rate, and promises a better coexistence between the surgeon and the robot. Previously, only one study addressing similar LOS issues has been presented, which utilised the Navigation Camera Assistance (NCA) system to move the camera in order to achieve the optimal LOS (Schaller, et al., 2011). However, as the registration of NCA is based on optical markers, it is not effective for general obstacles, such as surgical staff entering the workspace of the robot. Thus, our pilot study explores new possibilities of human-robot collaboration in the OR, and has wider application prospects.

From the experimental results, the performance of the system for slow obstacles is promising, significantly reducing the obstacle infringing depth. However, the performance for fast obstacles is currently relatively limited, as only objects inside the defined cone are considered in order to increase the speed of image processing. With faster imaging technology tracking moving objects in the whole environment, better motion plans can be made in advance to globally minimise the possibility of LOS interruption, thus achieving a more fluent and surgeon-centred workflow. 


\section{References}

Besl, P. J., \& McKay, N. D. (1992). Method for registration of 3-D shapes. Robotics-DL tentative (pp. 586-606). International Society for Optics and Photonics.

Goradia, V. K. (2014). Computer-assisted and robotic surgery in orthopedics: where we are in 2014. Sports medicine and arthroscopy review, 22(4), 202-205.

Karthik, K., Colegate-Stone, T., Dasgupta, P., Tavakkolizadeh, A., \& Sinha, J. (2015). Robotic surgery in trauma and orthopaedics. Bone Joint J, 97(3), 292-299.

Koenig, J. H., \& Hepinstall, M. S. (2015). Available Robotic Platforms in Partial and Total Knee Arthroplasty. Operative Techniques in Orthopaedics, 25(2), 85-94.

Lang, J. E., Mannava, S., Floyd, A. J., Goddard, M. S., Smith, B. P., Mofidi, A., \& Jinnah, R. H. (2011). Robotic systems in orthopaedic surgery. J Bone Joint Surg Br, 93(10), 1296-1299.

Langmann, B., Hartmann, K., \& Loffeld, O. (2012). Depth Camera Technology Comparison and Performance Evaluation. ICPRAM, 2, pp. 438-444.

Lea, J. T., Watkins, D., Mills, A., Peshkin, M. A., Kienzle, T. C., \& Stulberg, S. D. (1995). Registration and immobilization in robot-assisted surgery. Journal of image guided surgery, $1(2), 80-87$.

Maintz, J. A., \& Viergever, M. A. (1998). A survey of medical image registration, Medical image analysis. Medical image analysis, 2(1), 1-36.

Moors, E. (1920). On the reciprocal of the general algebraic matrix, abstract. Bull. Amer. Math. Soc, 26, 394-395.

Netravali, N. A., Shen, F., Park, Y., \& Bargar, W. L. (2013). A perspective on robotic assistance for knee arthroplasty. Advances in orthopedics.

Schaller, S., Strauss, G., Krinninger, M., Hurka, F., Hofer, M., Meixensberger, J., \& Lueth, T. C. (2011). Influence of an auto-motorised optical navigation camera to the surgical workflow in ENT surgery. Laryngo-rhino-otologie, 90(6), 353-357.

Sciavicco, L., \& Siciliano, B. (2012). Modelling and control of robot manipulators. Springer Science \& Business Media.

Sugano, N. (2013). Computer-assisted orthopaedic surgery and robotic surgery in total hip arthroplasty. Clinics in orthopedic surgery, 5(1), 1-9. 